\title{
Early parenteral nutrition in critically ill patients with short-term relative contraindications to early enteral nutrition: a full economic analysis of a multicenter randomized controlled trial based on US costs
}

This article was published in the following Dove Press journal:

ClinicoEconomics and Outcomes Research

20 July 2013

Number of times this article has been viewed

\section{Gordon S Doig \\ Fiona Simpson}

On behalf of the Early PN

Trial Investigators Group

Northern Clinical School Intensive Care Research Unit, University of Sydney, Sydney, NSW, Australia
Correspondence: Gordon S Doig Royal North Shore Hospital, Intensive Care Unit, Pacific Hwy, St Leonards, NSW, Australia 2065

$\mathrm{Tel}+6 \mathrm{I} 294632607$

Fax +6I 294632056

Email gdoig@med.usyd.edu.au
Purpose: The provision of early enteral (gut) nutrition to critically ill patients, started within 24 hours of injury or intensive care unit admission, is accepted to improve health outcomes. However, not all patients are able to receive early enteral nutrition. The purpose of the economic analysis presented here was to estimate the cost implications of providing early parenteral (intravenous) nutrition to critically ill patients with short-term relative contraindications to early enteral nutrition.

Materials and methods: From the perspective of the US acute care hospital system, a cost-minimization analysis was undertaken based on large-scale Monte Carlo simulation ( $N=1,000,000$ trials) of a stochastic model developed using clinical outcomes and measures of resource consumption reported in a 1,363-patient multicenter clinical trial combined with cost distributions obtained from the published literature. The mean costs of acute care attributable to each study group (early parenteral nutrition versus pragmatic standard care) and the mean cost difference between groups, along with respective $95 \%$ confidence intervals, were obtained using the percentile method.

Results and conclusion: The use of early parenteral nutrition in critically ill patients with short-term relative contraindications to early enteral nutrition may significantly and meaningfully reduce total costs of acute hospital care by US $\$ 3,150$ per patient ( $95 \%$ confidence interval US\$1,314 to US\$4,990). These findings were robust, with all sensitivity analyses demonstrating significant savings attributable to the use of early parenteral nutrition, including sensitivity analysis conducted using European cost data.

Keywords: intensive care, acute hospital care, intravenous nutrition, US acute hospital system

\section{Introduction}

The provision of early and appropriate nutrition support to patients during a critical illness is accepted to improve health outcomes, ${ }^{1}$ with the preponderance of the clinical evidence suggesting that most benefit can be obtained from the provision of early enteral (gut) feeding. ${ }^{2-4}$ Unfortunately, enteral feeding is often difficult to initiate early during critical illness, with multinational observational studies demonstrating that up to $45 \%$ of eligible critically ill patients do not have enteral feeding started within the timeframes recommended by international guidelines. ${ }^{5-7}$ 
In 2005, we conducted a systematic review of all clinical trials to evaluate the benefits attributable to enteral nutrition compared with parenteral (intravenous) nutrition in critical illness. ${ }^{8}$ We concluded that if early parenteral nutrition was provided to critically ill patients in whom early enteral nutrition could not be initiated, lives may be saved. Unfortunately, these same clinical trials also suggested that infectious complications might be increased if early parenteral nutrition was provided. To resolve the discordant results arising from the small trials published on this topic, we initiated a large-scale multicenter clinical trial (the Early Parenteral Nutrition [PN] Trial).

Details regarding the conduct and results of the Early PN Trial have been reported elsewhere., ${ }^{9,10}$ The purpose of this current paper is to report a full economic analysis based on the clinical results and measures of resource consumption reported in the Early PN Trial.

\section{Materials and methods}

\section{Context}

The Early PN Trial was a multicenter randomized controlled trial (RCT) conducted to determine whether adult critically ill patients with short-term relative contraindications to early enteral (gut) nutrition would benefit from the provision of early parenteral (intravenous) nutrition.

Within 24 hours of admission to an intensive care unit (ICU), critically ill patients unable to receive early enteral (gut) feeding due to a short-term relative contraindication were randomized to commence parenteral nutrition immediately or to receive pragmatic standard care. Standard care was defined pragmatically, not by study protocol, and allowed the attending clinician to select the route (gut or intravenous), starting rates, metabolic targets, and composition of nutrition to be provided to patients based on their ICU's current practice.

From October 2006 until June 2011, 1,363 critically ill patients were enrolled and randomized from the ICUs of 31 hospitals throughout Australia and New Zealand.

The provision of early parenteral nutrition did not alter the study's primary outcome, patient mortality at study day 60 $(0.0 \%$ covariate adjusted risk difference, $95 \%$ confidence interval (CI) from $-4.2 \%$ to $4.3 \%$ ); however, patients receiving early parenteral nutrition consumed less health care resources, as indicated by a reduction in need for invasive mechanical ventilation (MV; 1.1 fewer days, 95\% CI 0.3 to 1.8 ) and a reduction in duration of ICU stay ( 0.8 fewer days, $95 \%$ CI 0.0 to 1.5 fewer days). Patients randomized to the early parenteral nutrition arm of the clinical trial received a mean of 3.1 days (95\% CI 2.4 days to 4.0 days) more parenteral nutrition than standard care patients. There were no differences between randomized groups with regards to any other measures of resource consumption or health states (hospital stay, infection rates, antibiotic use, etc) or any suggestions of harm attributable to the use of early parenteral nutrition. Furthermore, no differential treatment effects were detected in a priori planned subgroup analysis based on nutritional status at baseline. ${ }^{10}$

\section{Type of economic evaluation}

In the context of the $95 \%$ CIs around the estimate of zero effect on day- 60 mortality rates, a cost-minimization analysis (CMA) was undertaken to compare total costs of care associated with the use of early parenteral nutrition with total costs of care associated with pragmatic standard care.

Total costs of care were estimated from measures of resource consumption reported in the Early PN Trial and costs obtained from the published literature using a stochastic model, with a large-scale Monte Carlo simulation undertaken to estimate total cost differences and 95\% CIs.

\section{Perspective and time horizon}

This CMA was conducted from the perspective of the US acute care hospital system. The time horizon of the study was the period from study enrollment until discharge from acute care hospital.

\section{Discounting/indexing of costs}

The US Department of Labor Consumer Price Index for Medical Consumers (CPI) was used to index published US costs to 2012 US funds based on the specific index rate reported for each year. ${ }^{11}$ In addition, a sensitivity analysis was conducted using a conservative index rate of $4.0 \% .^{12}$

All costs reported in this manuscript are indexed to 2012 US funds using the CPI, unless explicitly reported otherwise.

\section{Details concerning the delivery of the study intervention (early parenteral nutrition)}

At all participating sites, Early PN Trial intervention patients received standard parenteral nutrition from a ready-to-mix multi-chamber bag containing amino acids, glucose, lipids and electrolytes (Kabiven ${ }^{\circledR}$ G19\%, Fresenius Kabi Australia Pty Limited, Sydney, Australia). Complete product information is available online. ${ }^{13}$

Metabolic targets were set using the Harris-Benedict equation with appropriate adjustment factors (Table S1). Starting rates and daily rate increases were defined by two study PN delivery protocols (A and B), with Protocol B 
specifically designed for patients clinically suspected to be malnourished, who are known to be at risk of refeeding syndrome. Protocol B was the default protocol for patients with very low body mass index $\left(<17 \mathrm{~kg} / \mathrm{m}^{2}\right)$. Both PN delivery protocols reminded clinicians to provide vitamins, minerals, and trace elements daily, as clinically indicated. Protocol $\mathrm{B}$ made strong recommendations for the daily provision of vitamins, minerals, and trace elements to malnourished patients, who are known to be at risk of refeeding syndrome. See Table S2 for complete details regarding the study PN delivery protocols.

The study parenteral nutrition was a shelf-stable product that did not contain vitamins or trace elements at time of delivery to the study hospital, so it did not require refrigeration for storage. Delivery of the study parenteral nutrition to hospitals participating in the Early PN Trial was received by the nutrition department or study ICU and the product was stored in the ICU's storeroom until use. Pharmacy storage and handling were not required.

Guided by a detailed study protocol, the administration and delivery of the study parenteral nutrition did not require review or supervision by a parenteral nutrition team, nor were any additional biochemical tests required beyond those routinely conducted on a daily basis by the study ICU. Furthermore, only patients who were sick enough to already need a central venous line in situ at time of screening for eligibility into the trial were recruited to participate, thus delivery of early parenteral nutrition did not require de novo placement of a dedicated central line.

Whilst receiving the study intervention, if the patient's clinical team decided the patient required additional vitamins, minerals, or micronutrients (see Table S2), they were delivered via a separate infusion bag and not admixed with the study parenteral nutrition; as such, requirements for preparation of the study parenteral nutrition in a laminar flow environment were avoided. Complete details of the study intervention, such as the identification of eligible patients and calculation of caloric targets, are reported elsewhere. ${ }^{9,10}$

\section{Costs of study intervention (early parenteral nutrition)}

In the stochastic model, delivery of the study parenteral nutrition to patients randomized to receive early parenteral nutrition and the delivery of parenteral nutrition to standard care patients was costed at the level of the individual patient.

The cost of delivering 1 day of parenteral nutrition therapy in the US market was obtained from the publication by Turpin et al. ${ }^{14}$ Using a transaction level cost database from the Premier Healthcare Alliance, which covers more than 400 hospitals in the USA, Turpin et al identified a total of 44,358 patients from 194 hospitals who had at least one transactional level cost recorded for parenteral nutrition whilst in hospital. The costs for 1 day of parenteral nutrition therapy, including all additives (vitamins, minerals, trace elements, etc) and all fees, were reported as US\$186.92 for treatment with a ready-to-mix multi-chamber bag and US $\$ 272.40$ for a pharmacy compounded bag of parenteral nutrition.

Although the specific intervention delivered in the Early PN Trial involved the use of ready-to-mix multi-chamber bags of parenteral nutrition, to improve generalizability, we elected to blend the costs of a ready-to-mix multi-chamber bag and a pharmacy compounded bag, as blending resulted in a more conservative (higher) estimate of study intervention costs used in the CMA. For the purpose of this simulation, the mean cost of 1 day of parenteral nutrition was estimated at US\$229.66, with a standard deviation of US\$60.44, indexed from the original publication to 2012 US dollars using the CPI.

\section{Measures of acute care hospital resource consumption}

For each of the 1,363 patients enrolled into the Early PN Trial, recorded values for major measures of resource consumption demonstrating marginal differences between randomized groups were abstracted from the Early PN Trial database at the individual patient level. These measures of resource consumption included the patient's ICU length of stay, number of days of parenteral nutrition provided, whether or not the patient received invasive MV during their ICU stay and the primary type of patient population descriptor (ie, medical patient, surgical patient, or trauma patient).

\section{Costs of acute care whilst in the ICU}

Cost distributions for acute care whilst admitted to an ICU were obtained from the published literature. Dasta et al reported the mean daily costs of care from the perspective of the acute care hospital for patients admitted to an ICU using an administrative database composed of 51,009 ICU patients from 253 geographically diverse hospitals across the USA. ${ }^{15}$ This database, maintained by NDCHealth, contains patient charges recorded by operational billing systems and is regularly audited for accuracy. Costs were estimated using hospital-specific cost-to-charge ratios. Hospitals contributing to this study were considered representative of the larger US hospital population in terms of geographic location, bed number, and teaching status. 
Dasta et al's reported costs were significantly higher for the first 2 days of ICU admission compared to subsequent days, with significant differences also existing between major patient groups (medical patient, surgical patient, and trauma patient) and between patients who received invasive MV during their ICU stay compared with patients who did not receive MV. Table 1 presents the relevant cost distribution matrix abstracted from Dasta et al's study.

\section{Structure of the stochastic cost model and large-scale Monte Carlo simulation}

Post-randomization costs of care were estimated using a stochastic model based on the sum of daily cost components, modeled using the gamma distribution with mean $\mu$ and shape $\alpha$, where $\alpha=\mu^{2} / \sigma^{2}$. For example, the costs of acute care for a trauma patient who received $\mathrm{MV}$, required 3 days of care in an ICU, and received 2 days of parenteral nutrition would be estimated as the sum of five randomly generated gamma distributed costs: Day 1 ICU stay $G$ (US $\$ 15,625$, US $\$ 11,955$ ) plus day 2 ICU stay $G$ (US\$7,414, US\$6,683) plus day 3 ICU stay $G$ (US\$5,880, US\$5,750) plus 1 day of parenteral nutrition $G$ (US\$229.66, US\$60.44) plus day 2 of parenteral nutrition $G$ (US\$229.66, US\$60.44), where $G$ (mean, standard deviation). If a patient was enrolled into the Early PN Trial on day 2 of their ICU stay, costing was begun with ICU day 2 . For those patients enrolled on ICU day 2, day 1 ICU costs would be assigned as zero. Daily costs for each major patient type abstracted from Dasta et $\mathrm{al}^{15}$ are reported in Table 1.

For each of the 1,363 patients enrolled into the Early PN Trial, costs were estimated for $\mathrm{N}=1,000,000$ episodes of care to generate stable estimates of costs and CIs. The CMA was based on the net differences in costs between the $1,000,000$ simulated clinical trial groups.

All simulations were conducted using PC SAS (v 9.2, SAS Institute Inc, Cary, NC, USA).

\section{Calculation of the mean costs and $95 \% \mathrm{Cls}$}

The mean costs of acute care attributable to each study group (early parenteral nutrition versus standard care) and the mean cost difference between groups, along with the respective $95 \%$ CIs, were obtained using the percentile method. As opposed to bootstrapping, which requires resampling and typically uses fewer trials $(\mathrm{N}=1,000)$, the percentile method does not require correction for bias when applied to large-scale simulations, which typically use more trials ( $N>250,000)$ with no resampling. ${ }^{16}$

\section{Sensitivity analyses}

Three sensitivity analyses were planned before conducting the primary CMA:

1. The primary CMA analysis was rerun using normal distributional assumptions for the generated cost data, instead of gamma distributional assumptions.

2. The primary CMA analysis was rerun using a conservative discount of $4 \%$ per annum, instead of discounting according to the CPI.

3. The primary CMA analysis was rerun using published daily costs of ICU care and study intervention costs for the European market, instead of published US costs. Based on a micro-costing study conducted in the Netherlands, the average total cost of one ventilated ICU day has been reported as (mean [standard deviation]) €2,644 (€2,502) and for an unventilated ICU day as $€ 2,081(€ 1,914)$, indexed to 2012 Euros at $4 \%$ per annum. ${ }^{17,18}$ The European costs for the study intervention were obtained from an economic analysis conducted in Belgium. ${ }^{19}$ The marginal cost increase attributable to the provision of 1 day of parenteral nutrition to a critically ill ICU patient was reported as $€ 102$ per day (indexed to 2012 funds at $4 \%$ per annum), which was reported to include acquisition (purchase costs), additional vitamins, minerals, administration of the parenteral nutrition, and the cost of monitoring.

\section{Results}

The 1,000,000-trial Monte Carlo simulation required 1 hour 17 minutes to execute on a $5.1 \mathrm{GHz}$ Intel $3930 \mathrm{~K}$ processor (Intel, Santa Clara, CA, USA) with 64 GB of memory and six Intel 520 series solid-state drives in RAID 0 on an LSI 9265 SCSI controller. The 1,363 patients in the Early PN Trial

Table I Matrix of the distributions of daily costs of care whilst admitted to the intensive care

\begin{tabular}{|c|c|c|c|c|c|c|}
\hline & \multicolumn{2}{|c|}{ Medical patients } & \multicolumn{2}{|l|}{ Surgical patients } & \multicolumn{2}{|l|}{ Trauma patients } \\
\hline & Received MV & No $M V$ received & Received MV & No $M V$ received & Received MV & No $M V$ received \\
\hline Day I & $\$ 8,|4|(\$ 5,584)$ & $\$ 5,357(\$ 5,584)$ & $\$ 20,582(\$ 14,3 \mid 9)$ & $\$ 9,916$ (\$14,319) & $\$ 15,625(\$ 11,955)$ & $\$ 9,062(\$ 11,955)$ \\
\hline Day 2 & $\$ 6,535(\$ 4,678)$ & $\$ 4,783(\$ 4,678)$ & $\$ 7,726(\$ 6,977)$ & $\$ 5,050(\$ 6,977)$ & $\$ 7,414(\$ 6,683)$ & $\$ 4,968(\$ 6,683)$ \\
\hline Day 3 plus & $\$ 5,703(\$ 4,666)$ & $\$ 4,26 \mid(\$ 4,666)$ & $\$ 6,627(\$ 5,624)$ & $\$ 4,765(\$ 5,624)$ & $\$ 5,880(\$ 5,750)$ & $\$ 4,64 \mid(\$ 5,750)$ \\
\hline
\end{tabular}

Notes: Mean costs (standard deviation); indexed to 2012 US dollars. Costs of care whilst admitted to the intensive care unit were abstracted from Dasta JF et al. 15 Abbreviation: MV, mechanical ventilation. 
consumed 11,424 ICU days requiring the stochastic estimation of $11,424,000,000$ cost-days $(11,424$ days $\times 1,000,000$ trials) resulting in a $107 \mathrm{~GB}$ data file.

Complete details of the Early PN Trial patient population are reported elsewhere. ${ }^{10}$ Specifically relevant to this simulation, 33\% $(443 / 1,363)$ of patients were classified as medical, $64 \%(880 / 1,363)$ as surgical, and 3\% $(40 / 1,363)$ as trauma. Fifteen percent $(206 / 1,363)$ of patients never received MV during their ICU stay. Forty-four percent $(593 / 1,363)$ of patients were enrolled into the Early PN Trial on day 1 of their ICU stay, $56 \%(769 / 1,363)$ were enrolled on day 2 , and $0.07 \%(1 / 1,363)$ of patients were enrolled on day 3 .

To assess the accuracy of the simulation-generated cost structure, Table 2 presents the daily costs of acute care generated by the stochastic model, based on the cost matrix abstracted from Dasta et $\mathrm{al}^{15}$ (see Table 1) under gamma distributional assumptions by a 250,000-trial Monte Carlo simulation for the first 3 days of ICU stay for each main patient group.

\section{Primary CMA analysis: costs indexed to 2012 US dollars using the CPI, gamma distribution}

Using the percentile method, the mean cost of ICU care for patients randomly allocated to receive standard care was US\$58,923 per patient, with a 95\% CI of US\$57,631 to US\$60,239, whereas the mean cost of care for patients randomly allocated to receive early parenteral nutrition was US\$55,772 per patient, with a $95 \%$ CI of US\$54,488 to US\$57,082.

The simulation-estimated CMA revealed a US\$3,150 saving per patient in favor of early parenteral nutrition use, with a $95 \%$ CI of US\$1,314 to US\$4,990 saving per patient.

\section{Sensitivity analyses}

1. Under normal distributional assumptions, the mean cost difference was a US\$3,150 saving per patient in favor of early parenteral nutrition use, with a $95 \%$ CI of US $\$ 1,312$ to US\$4,988 in savings per patient.
2. Indexed at $4 \%$ per annum, the mean cost difference was a US\$3,036 saving per patient, in favor of early parenteral nutrition use (95\% CI US\$1,243 to US\$4,826 of savings per patient).

3. Using European ICU and study intervention costs, the mean cost difference was a $€ 1,854$ saving per patient in favor of early parenteral nutrition use $(95 \% \mathrm{CI} € 1,103$ to $€ 2,605$ of savings per patient). At current exchange rates (1 EUR $=1.30140 \mathrm{USD}$, at mid-market rates on April 24, 2013 at $10.21 \mathrm{pm}$ Coordinated Universal Time), this equates to US\$2,412 of savings in favor of early parenteral nutrition use (95\% CI US\$1,435 to US\$3,389).

\section{Discussion}

We undertook a full economic analysis to assess the cost implications of providing early parenteral nutrition to adult critically ill patients with short-term relative contraindications to early enteral nutrition. Measures of clinical outcomes and health care resource consumption were obtained from a multicenter clinical trial (the Early PN Trial). Costs of care and costs of providing early parenteral nutrition were obtained from the published literature. Large-scale Monte Carlo simulation of a stochastic cost model revealed the provision of early parenteral nutrition might reduce the overall cost of care by US $\$ 3,150$ per patient ( $95 \%$ CI US $\$ 1,314$ to US\$4,990). These findings were robust, with all sensitivity analyses demonstrating significant savings attributable to the use of early parenteral nutrition, including the sensitivity analysis conducted using European cost data.

Compared with previous economic analyses assessing the costs of nutrition therapy, which have largely been based on evidence of effectiveness of questionable methodological quality and taken a narrow focus on upfront acquisition costs, ${ }^{20}$ our CMA is based on the results of a multicenter clinical trial conducted in a focused patient population ${ }^{10}$ using published costs obtained from comprehensive databases with a broad perspective. ${ }^{14,15}$ Although our findings of significant and

Table 2 Matrix of the distributions of daily costs of care whilst admitted to the intensive care unit generated by a 250,000-trial Monte Carlo simulation

\begin{tabular}{|c|c|c|c|c|c|c|}
\hline & \multicolumn{2}{|c|}{ Medical patients } & \multicolumn{2}{|l|}{ Surgical patients } & \multicolumn{2}{|l|}{ Trauma patients } \\
\hline & Received MV & Never MV & Received MV & Never MV & Received MV & Never MV \\
\hline \multirow[t]{2}{*}{ Day I } & $\$ 8,|4|(\$ 5,585)$ & $\$ 5,353(\$ 5,578)$ & $\$ 20,58 \mid(\$|4,3| 7)$ & $\$ 9,9 \mid 7$ (\$14,3|5) & $\$ 15,627(\$ 1 \mid, 948)$ & $\$ 9,085(\$ 12,006)$ \\
\hline & $N=39,250,000$ & $N=5,750,000$ & $\mathrm{~N}=81,750,000$ & $N=16,500,000$ & $\mathrm{~N}=4,750,000$ & $N=250,000$ \\
\hline \multirow[t]{2}{*}{ Day 2} & $\$ 6,534(\$ 4,677)$ & $\$ 4,779(\$ 4,676)$ & $\$ 7,725(\$ 6,976)$ & $\$ 5,048(\$ 6,972)$ & $\$ 7,416(\$ 6,684)$ & $\$ 4,966(\$ 6,699)$ \\
\hline & $N=90,750,000$ & $N=14,000,000$ & $N=175,750,000$ & $N=31,500,000$ & $N=9,750,000$ & $N=250,000$ \\
\hline \multirow[t]{2}{*}{ Day 3 plus } & $\$ 5,702(\$ 4,665)$ & $\$ 4,262(\$ 4,668)$ & $\$ 6,627(\$ 5,624)$ & $\$ 4,764(\$ 5,625)$ & $\$ 5,882(\$ 5,750)$ & $\$ 4,626(\$ 5,739)$ \\
\hline & $\mathrm{N}=82,500,000$ & $N=11,750,000$ & $N=151,500,000$ & $N=19,750,000$ & $\mathrm{~N}=8,250,000$ & $N=250,000$ \\
\hline
\end{tabular}

Notes: Mean costs (standard deviation); 2012 US dollars.

Abbreviations: MV, mechanical ventilation; N, number of cost estimates simulated and pooled (iterations). 
substantial cost savings may appear to conflict with a recent economic analysis reporting increased costs attributable to parenteral nutrition, ${ }^{19}$ this previously published economic analysis addressed a different clinical indication for parenteral nutrition than did our CMA. That study, by Vanderheyden et $\mathrm{al}^{20}$, assessed the financial consequences of administering additional parenteral nutrition to critically ill patients who were already able to receive enteral nutrition, ${ }^{21}$ whereas our CMA addressed the financial consequences of administering parenteral nutrition to patients who were unable to receive early enteral nutrition due to short-term relative contraindications. ${ }^{10}$

A series of a priori defined sensitivity analyses was undertaken to explore alternate decisions regarding major assumptions behind the primary CMA. Each of these sensitivity analyses concurs with the primary CMA, demonstrating significantly reduced costs associated with early parenteral nutrition use. Use of the CPI to index reported costs to 2012 US funds controlled for realistic cost increases over time, and led to an average index rate of $4.2 \%$ per annum, only slightly higher than the conservative sensitivity analysis index rate of $4.0 \%$. Additionally, the results obtained by the sensitivity analysis under normal distributional cost assumptions were essentially identical to the primary CMA results, conducted under the gamma distribution. It is possible that distributional assumptions are more important when conducting smaller simulations but become moot in large-scale simulations ( $N=1,000,000$ trials). Furthermore, the sensitivity analysis conducted under European cost assumptions also reported significant costs savings, which supports the primary CMA results based on US costs.

Although estimates of savings obtained by the sensitivity analysis conducted with European costs may appear lower than US savings, because the $95 \%$ CIs of the two estimates overlap, we cannot claim that estimates of cost savings differ significantly between the two different health care systems. Although it is commonly accepted that the US spends more on health care as a percent of its gross domestic product than any other country $(17.9 \%$ of gross domestic product in $2011^{22}$ ), the apparent difference between estimates of US and European cost savings reported in this CMA may be due to different cost-accounting methods used in the US versus Europe. Whereas the perspective taken by Dasta et $\mathrm{al}^{15}$ in estimating US costs was broad and included all hospital costs incurred whilst patients were cared for in an ICU, the perspective taken by Tan et al ${ }^{18}$ to estimate European costs was slightly narrower. For example, although Tan et al explicitly reported accounting for costs of consultation time for nonICU clinicians consulting on patients in the ICU, they did not report whether services delivered to critically ill patients by departments outside the ICU (eg, operating theatre expenses) were fully accounted for.

Indeed, many costing studies from Europe report the daily cost of ICU care as considerably lower than US-based studies; however, the European studies often employ a very restricted perspective, frequently reporting only direct costs of ICU care, ${ }^{23}$ treating the ICU as a cost center within the acute care hospital. The economic assessment of competing health care alternatives delivered whilst a patient is cared for in an ICU may require a broader perspective. For example, technologies that increase direct costs to the ICU (eg, lease of an air suspension bed to prevent pressure ulcers, paid from the ICU budget) may prove cost-effective only when reduced costs to departments outside the ICU are considered (eg, reduced need for operating theatre time to conduct debridement surgery for Stage 3 and 4 pressure ulcers, paid from the surgery department budget). ${ }^{24}$

\section{Strengths and limitations}

In addition to providing costs from the perspective of the acute care hospital, which include services offered by departments outside of the ICU, the cost matrix reported by Dasta et al (Table 1) allows for accurate stochastic modeling because specific cost distributions can be assigned to specific patient groups (ventilated, medical, surgical, trauma) for each ICU day. ${ }^{15}$ Furthermore, these US cost estimates, and the cost estimates for parenteral nutrition, were generated from robust databases containing tens of thousands of transactions from hundreds of hospitals. ${ }^{14,15}$

Although the European micro-costing study by Tan et al does provide cost estimates for ventilated and unventilated ICU patient-days, it does not allow for accounting by patient type and was conducted in only one hospital from a narrower perspective. ${ }^{18}$ Furthermore, as a consumer price index for medical consumers was not readily available for the Netherlands ${ }^{25}$ to allow comparability with the primary US-based CMA, the costs reported by Tan et a ${ }^{18}$ were indexed to 2012 funds using the conservative rate of $4 \%$.

Consideration of European financial consequences was not a central objective of this paper. The European-based analysis was undertaken in the context of the primary CMA, as part of a sensitivity analysis. A more thorough analysis, using more comprehensive cost estimates obtained from a broader perspective indexed using a harmonized European consumer price index for medical consumers may be required to draw firm conclusions regarding European costs.

\section{Conclusion}

We conducted a CMA based on the clinical outcomes and measures of resource consumption reported in a multicenter 
RCT published in the Journal of the American Medical Association, with costs obtained from comprehensive and validated databases, reported from the broad perspective of the acute care hospital system. Within the context of the clinical question addressed by the underlying RCT, we found the use of parenteral nutrition in critically ill patients with short-term relative contraindications to enteral nutrition may significantly and meaningfully reduce total cost of care.

\section{Acknowledgments}

Study Management Committee: Gordon S Doig (Chair), Fiona Simpson, Elizabeth A Sweetman, Simon R Finfer, D Jamie Cooper, Philippa T Heighes, Andrew R Davies, Michael O'Leary, Tom Solano and Sandra Peake. PN protocol sub-committee: Gordon S Doig (Chair), Fiona Simpson, Michael O'Leary. Infectious complications sub-committee: Gordon S Doig (Chair), Tom Solano, Fiona Simpson. Data Quality and Management: Jennifer L Hannam (Northern Clinical School Intensive Care Research Unit, University of Sydney, Australia). Statistical analysis: Gordon S Doig. Independent Data Safety and Monitoring Committee: John Moran (Chair, Dept of Intensive Care, The Queen Elizabeth Hospital, Adelaide, Australia), Petra Graham (Dept of Statistics, Macquarie University, Sydney, Australia) and Andrew Bersten (Dept of Critical Care Medicine, Flinders University, Adelaide, Australia).

Early PN Trial Contributing Sites and Site Investigators, alphabetical by site: Auckland City Hospital, New Zealand: Jodi Brown, Heidi Buhr, Vicki Cochrane, Michelle Eccleston, Eileen Gilder, Shay McGuiness, Rachael Parke, Anna Whitley. Austin Hospital, Victoria, Australia: Rinaldo Bellomo, Glenn Eastwood, Donna Goldsmith, Inga Mercer, Kim O'Sullivan, Leah Peck, Helen Young. Bendigo Hospital, Victoria, Australia: Catherine Boschert, John Edington, Jason Fletcher, Gary Koch, Mainak Majumdar, Tracey Shard, Julie Smith. Blacktown Hospital, New South Wales, Australia: Kalpesh Gandhi, Kiran Nand, Treena Sara. Box Hill Hospital, Victoria, Australia: David Charlesworth, Suzanne Eliott, David Ernest, Angela Hamilton (deceased), Belinda Howe, Inga Mercer, Sam Radford, Jaspreet Sidhu. Cabrini Hospital, Victoria, Australia: Jonathon Barrett, Felicity Hawker, MariaGrazia de Luca. Calvary Mater Hospital Newcastle, New South Wales, Australia: Irene Bailey, Jorge Brieva, Katrina Ellem. Campbelltown Hospital, New South Wales, Australia: Gillian Bishop, Olivia Mulligan, Ray Eckhardt. Concord Hospital, New South Wales, Australia: David Milliss, Helen Wong. Dandenong Hospital, Victoria, Australia: Subhash Arora, Michael Buist, Bridget O’Bree, Kate Shepherd, Susan Van Dyk. Frankston Hospital, Victoria, Australia: Sharon Allsop,
Subhash Arora, John Botha, Himangsu Gangopadhyay, David Lewis, Naomi Pratt, Fiona Turnbull, Jodi Vuat. Geelong Hospital, Victoria, Australia: Allison Bone, Claire Cattigan, Tania Elderkin, Melissa Fraser, Anne Kilmonth, Neil Orford, Tania Salerno. Gold Coast Hospital, Queensland, Australia: Alan Spencer, Mandy Tallott, Rosemary Whitbread. Gosford Hospital, New South Wales, Australia: Rob Cameron, Sheridan Hatter, Jackie Hyslop, Peter Rye. John Flynn Private Hospital, Queensland, Australia: Robin Holland, Roslyn van der Vooren. John Hunter Hospital, New South Wales, Australia: Elise Crowfoot, Miranda Hardie, Peter Harrigan, Sam Jenkins. Liverpool Hospital, New South Wales, Australia: Deepak Bhonagiri, Sharon Micallef, Michael Parr. Lyell McEwin Hospital, South Australia, Australia: Rajaram Ramadoss, Josette Wood, Julie Zuppa. Middlemore Hospital, New Zealand: Marilyn Beggs, Peter Dzendrowskyj, Chantal Hogan, Judy Tai, Anna Tilsley, Tony Williams. Monash Medical Centre, Victoria, Australia: Jonathon Barrett, Sue Burton, Tim Crozier, Pauline Galt, Ainsley Gillies, Rebecca Ioannidis, Marnie Reilly, Carly Thornhill. Nepean Hospital, New South Wales, Australia: Cheryl Cuzner, Rebecca Gresham, Larissa Hoyling, Tony Maclean, Maria Nikas, Phoebe Palejs, Ian Seppelt, Leonie Weisbrodt, Sarah Whereat. Royal North Shore Hospital, New South Wales, Australia: Anthony Delaney, Gwen Hickey. Royal Hobart Hospital, TAS: David Cooper, Kathryn Marsden, Rick McAllister, Ram Sistla, Andrew Turner. St George Hospital, New South Wales, Australia: Vanessa Dhiacou, Deb Inskip, Theresa Jacques, Alina Jovanovska, Michael O'Leary, Rebecca Sidoli. St Vincent's Hospital Melbourne, Victoria, Australia: Nicole Groves, Jenny Holmes, John Santamaria, Roger Smith, Antony Tobin. St Vincent's Hospital Sydney, New South Wales, Australia: Jeff Breeding, Priya Nair, Claire Reynolds, Karen Storer. Sydney Adventist Hospital, New South Wales, Australia: Roger Harris, Linley Shields, Hui (Whay) Yang. The Prince of Wales Hospital, New South Wales, Australia: Frances Bass, Michelle Campbell, Pam Edhouse, Naomi Hammond, Maryam Sana, Yahya Shehabi, Victoria Stockdale, Barb Trytko. The Queen Elizabeth Hospital, South Australia, Australia: Catherine Kurenda, Sandra Peake, Patricia Williams. Wellington Hospital, New Zealand: Lynn Andrews, Dick Dinsdale, Peter Hicks, Diane Mackle. Wollongong Hospital, New South Wales, Australia: Michael Davis, Michelle Gales, Francisco Hill, Bronwyn Johnson, Adam Purdon, Martin Sterba, Renee Xu.

\section{Disclosure}

This full economic analysis was supported by a peer-reviewed academic grant from the Australian National Health and 
Medical Research Council (Project Grant Number 402643). As a peer review funding body, the Australian National Health and Medical Research Council provided constructive comments on the study design but played no role in the conduct of the study; the collection, management, analysis, and interpretation of data; or the preparation, review, or approval of the manuscript. The interpretation and reporting of the data are the sole responsibilities of the authors.

Dr Doig has received academic research grants from Fresenius Kabi Deutschland GmbH, Baxter Healthcare Pty Ltd, and Nestle Nutrition; consulting fees from Nestle Nutrition, Fresenius Kabi Deutschland $\mathrm{GmbH}$, and Baxter Healthcare Pty Ltd; speaker's honoraria from Nestle Nutrition, Baxter Healthcare Pty Ltd, and Fresenius Kabi Deutschland GmbH; and travel support from Fresenius Kabi Deutschland $\mathrm{GmbH}$, Baxter Healthcare Pty Ltd, and Nestle Nutrition. Ms Simpson has received academic research grants from Fresenius Kabi Deutschland GmbH and Baxter Healthcare Pty Ltd, speaker's honoraria from Fresenius Kabi Pty Ltd, and travel support from Fresenius Kabi Deutschland GmbH and Baxter Healthcare Pty Ltd. The authors declare no other conflicts of interest in this work.

\section{References}

1. Martin CM, Doig GS, Heyland DK, Morrison T, Sibbald WJ; Southwestern Ontario Critical Care Research Network. Multicentre, cluster-randomized clinical trial of algorithms for critical-care enteral and parenteral therapy (ACCEPT). CMAJ. 2004;170(2):197-204.

2. Doig GS, Heighes PT, Simpson F, Sweetman EA. Early enteral nutrition reduces mortality in trauma patients requiring intensive care: a metaanalysis of randomised controlled trials. Injury. 2011;42(1):50-56.

3. Doig GS, Heighes PT, Simpson F, Sweetman EA, Davies AR. Early enteral nutrition, provided within $24 \mathrm{~h}$ of injury or intensive care unit admission, significantly reduces mortality in critically ill patients: a meta-analysis of randomised controlled trials. Intensive Care Med. 2009;35(12): 2018-2027.

4. Heyland DK, Dhaliwal R, Drover JW, Gramlich L, Dodek P; Canadian Critical Care Clinical Practice Guidelines Committee. Canadian clinical practice guidelines for nutrition support in mechanically ventilated, critically ill adult patients. JPEN J Parenter Enteral Nutr. 2003;27(5): 355-373.

5. Heyland DK, Heyland RD, Cahill NE, et al. Creating a culture of clinical excellence in critical care nutrition: the 2008 "Best of the Best" award. JPEN J Parenter Enteral Nutr. 2010;34(6):707-715.

6. McClave SA, Martindale RG, Vanek VW, et al; A.S.P.E.N. Board of Directors; American College of Critical Care Medicine; Society of Critical Care Medicine. Guidelines for the Provision and Assessment of Nutrition Support Therapy in the Adult Critically Ill Patient: Society of Critical Care Medicine (SCCM) and American Society for Parenteral and Enteral Nutrition (A.S.P.E.N.). JPEN J Parenter Enteral Nutr. 2009;33(3):277-316.

7. Kreymann KG, Berger MM, Deutz NE, et al; DGEM (German Society for Nutritional Medicine), Ebner C, Hartl W, Heymann C, Spies C; ESPEN (European Society for Parenteral and Enteral Nutrition). ESPEN Guidelines on Enteral Nutrition: Intensive care. Clin Nutr. 2006;25(2):210-223.

8. Simpson F, Doig GS. Parenteral vs enteral nutrition in the critically ill patient: a meta-analysis of trials using the intention to treat principle. Intensive Care Med. 2005;31(1):12-23.
9. Doig GS, Simpson F, Sweetman EA, Heighes PT; Early PN Trial Management Committee. Statistical Analysis Plan for a Multi-centre Randomised Controlled Trial: Early Parenteral Nutrition vs Standard Care in Patients not Expected to be Fed within 24 h of ICU Admission. Sydney: University of Sydney; 2011. Available from: http://www. evidencebased.net/files/EarlyPN_SAP.pdf. Accessed June 17, 2013.

10. Doig GS, Simpson F, Sweetman EA, et al; Early PN Investigators of the ANZICS Clinical Trials Group. Early parenteral nutrition in critically ill patients with short-term relative contraindications to early enteral nutrition: a randomized controlled trial. JAMA. 2013;309(20):2130-2138.

11. US Bureau of Labor Statistics. Consumer Price Index Detailed Report: Medical Care Consumer Price Index; All Urban Consumers. 2013. Available from: http://data.bls.gov/pdq/SurveyOutputServlet. Accessed June 27, 2013.

12. Alban A, Gyldmark M, Pedersen AV, Søgaard J. The Danish approach to standards for economic evaluation methodologies. Pharmacoeconomics. 1997;12(6):627-636.

13. Kabiven ${ }^{\circledR}$ G19\% [product information]. Pymble: Fresenius Kabi Australia Pty Limited. Available from: http:/www.fresenius-kabi.com.au/ files/Kabiven_G19_PI.pdf. Accessed June 11, 2013.

14. Turpin RS, Canada T, Liu FX, Mercaldi CJ, Pontes-Arruda A, Wischmeyer P. Nutrition therapy cost analysis in the US: pre-mixed multi-chamber bag vs compounded parenteral nutrition. Appl Health Econ Health Policy. 2011;9(5):281-292.

15. Dasta JF, McLaughlin TP, Mody SH, Piech CT. Daily cost of an intensive care unit day: the contribution of mechanical ventilation. Crit Care Med. 2005;33(6):1266-1271.

16. Greenland S. Interval estimation by simulation as an alternative to and extension of confidence intervals. Int J Epidemiol. 2004;33(6): 1389-1397.

17. Al MJ, Hakkaart L, Tan SS, Bakker J. Cost-consequence analysis of remifentanil-based analgo-sedation vs conventional analgesia and sedation for patients on mechanical ventilation in the Netherlands. Critical Care. 2010;14(6):R195.

18. Tan SS, Hakkaart-van Roijen L, Al MJ, et al. Review of a large clinical series: a microcosting study of intensive care unit stay in the Netherlands. J Intensive Care Med. 2008;23(4):250-257.

19. Vanderheyden S, Casaer MP, Kesteloot K, et al. Early versus late parenteral nutrition in ICU patients: cost analysis of the EPaNIC trial. Critical Care. 2012;16(3):R96. Epub ahead of print.

20. Pritchard C, Duffy S, Edington J, Pang F. Enteral nutrition and oral nutrition supplements: a review of the economics literature. JPEN J Parenter Enteral Nutr. 2006;30(1):52-59.

21. Casaer MP, Hermans G, Wilmer A, Van den Berghe G. Impact of early parenteral nutrition completing enteral nutrition in adult critically ill patients (EPaNIC trial): a study protocol and statistical analysis plan for a randomized controlled trial. Trials. 2011;12:21.

22. World Bank. Health expenditure, total (\% of GDP) [web page on the Internet; data from World Health Organization National Health Account database]. Washington DC: World Bank; nd. Available from: http:// data.worldbank.org/indicator/SH.XPD.TOTL.ZS. Accessed April 30, 2013.

23. Tan SS, Bakker J, Hoogendoorn ME, et al. Direct cost analysis of intensive care unit stay in four European countries: applying a standardized costing methodology. Value Health. 2012;15(1):81-86.

24. Inman KJ, Sibbald WJ, Rutledge FS, Clark BJ. Clinical utility and cost-effectiveness of an air suspension bed in the prevention of pressure ulcers. JAMA. 1993;269(9):1139-1143.

25. de Haan J, Kleima F. Medical Care Price and Quantity Indexes: Dutch Practice and Unresolved Issues. Paper prepared for the Ninth Meeting of the International Working Group on Price Indices (Ottawa Group), London, May 14-16, 2006. Netherlands: Statistics Netherlands; 2006. Available from: http://www.ottawagroup.org/Ottawa/ottawagroup.nsf/ home/Meeting+9/\$file/2006 9th Meeting - van Haren and Kleima and de Haan - The CPI and the new Dutch Health Care System.pdf. Accessed June 17, 2013. 


\section{Supplementary tables}

Table SI Harris-Benedict equations and adjustment factors used by study website (www.EvidenceBased.net/EarlyPN)

\section{For males}

Target metabolic needs $(\mathrm{kcals} /$ day $)=[66.5+(13.75 \times \mathrm{Wt})+(5.003 \times \mathrm{Ht})-(6.775 \times \mathrm{Age})] \times$ adjustment factor

\section{For females}

Target metabolic needs $(\mathrm{kcals} / \mathrm{day})=[655 . \mathrm{I}+(9.563 \times \mathrm{Wt})+(\mathrm{I} .85 \times \mathrm{Ht})-(4.676 \times \mathrm{Age})] \times$ adjustment factor $\mathrm{Wt}=$ weight in $\mathrm{kg}$

$\mathrm{Ht}=$ height in $\mathrm{cm}$

Age $=$ Age in years

Adjustment factors (most severe was selected)

Other, not listed below $<$ value="I.2" $>$

- Any other problem, not listed below.

Infection, mild $<$ value="I.3" $>$

- Ex mild skin, line or surgical wound infection. Local redness, heat and swelling but no systemic signs.

Operation, minor $<$ value="I.3" $>$

- Any surgical procedure that does not require general anesthesia or respiratory support.

Operation, major $<$ value="I.35" $>$

- Any surgical procedure that does require general anesthesia or respiratory support.

Infection, peritonitis (non-septic) < value="I.35" $>$

- Peritonitis based on visual inspection or culture. Patient does not have systemic signs of sepsis.

Cancer $<$ value="I.35" $>$

- Patient is known to have an active tumor. May or may not be undergoing active or palliative treatment.

Trauma, single fracture (skeletal) < value="I.4">

- Patient has trauma resulting in a single skeletal fracture of any bone except long bones.

Infection, moderate $<$ value="I.45" $>$

- Infections that would normally require ICU admission for treatment. Ex Community acquired pneumonia, Ventilator Associated Pneumonia.

Trauma, single long-bone fracture $<$ value="I.45" $>$

- Trauma with a fracture to a long bone (femur, humerus, tibia, fibula, radius and ulna).

Trauma, multiple fractures $<$ value="I.5" $>$

- Trauma with multiple fractures to any bones, including at least one long bone.

Trauma, blunt with or without fractures $<$ value="I.6" $>$

- Blunt trauma, such as a motor vehicle crash and fall from height. Includes Penetrating trauma.

Infection, severe $<$ value="I.65" $>$

- Any infection, or suspected infection, that expresses itself systemically as sepsis.

Burns, less than or equal to $20 \%$ TBSA $<$ value="I.7" $>$

- Chemical or thermal burns to less than $20 \%$ of total body surface area.

Malnourished (high risk of refeeding syndrome) $<$ value=" 0.85 " $>$

- Body mass index of less than 17 or history and physical exam consistent with malnourishment or high risk of malnourishment. Based on clinical grounds decided by attending clinician.

Notes: Harris-Benedict calculated targets were capped at $35 \mathrm{kcal} / \mathrm{kg} /$ day and obese patients $\left(\mathrm{BMI} \geq 30 \mathrm{~kg} / \mathrm{m}^{2}\right)$ used ideal body weight $\left(\mathrm{BMI}=2 \mathrm{l} \mathrm{kg} / \mathrm{m}^{2}\right)$ in all Harris-Benedict calculations.

Abbreviations: kcals, kilocalories; Wt, weight in kilograms; $\mathrm{Ht}$, height in centimeters; kg, kilograms; cm, centimeters; Ex, example; ICU, intensive care unit; TBSA, total burn surface area. 
Table S2 PN (parenteral nutrition) Protocols

Study PN Protocol A: all early PN patients except malnourished

Feeding day I (first 24 hours of PN)

- Commence Kabiven G19\% at $60 \mathrm{~mL} / \mathrm{hr}$ (or goal rate, whichever is lower).

- Consider trace element, mineral and vitamin needs as clinically appropriate.

Feeding day 2 (second 24 hours of PN)

- Increase Kabiven G19\% to $80 \mathrm{~mL} / \mathrm{hr}$ (or goal rate, whichever is lower).

- Consider trace element, mineral and vitamin needs as clinically appropriate.

Feeding day 3 (next 24 hours)

- Increase Kabiven G19\% to goal rate, as appropriate.

- Consider trace element, mineral and vitamin needs, as clinically appropriate.

- Recommend trialing enteral/oral nutrition, if clinically appropriate.

- Once the patient tolerates $\geq 475 \mathrm{kcal} /$ day EN, complete remainder of 24 hour Kabiven infusion and do not hang another bag.

- If patient tolerates any oral caloric intake from food, complete remainder of 24 hour Kabiven infusion and do not hang another bag.

Feeding day 4 (next 24 hours) plus all additional days after day 4

- May switch to parenteral nutrition solution tailored to patient's specific clinical needs. Goals not to exceed $25-35 \mathrm{kcal} / \mathrm{kg}$ and I.0-1.5 g protein/kg.

- Consider long term needs regarding trace element, mineral and vitamins as clinically appropriate.

- Recommend trialing enteral/oral nutrition, if clinically appropriate.

- Once the patient tolerates $\geq 475 \mathrm{kcal} /$ day EN, complete remainder of 24 hour Kabiven infusion and do not hang another bag.

- If patient tolerates any oral caloric intake from food, complete remainder of 24 hour Kabiven infusion and do not hang another bag. Insulin/glucose protocol: early PN patients

If glucose levels exceed $10 \mathrm{mmol} / \mathrm{L}$ an insulin infusion should be commenced and titrated to achieve peak serum glucose levels of $<10 \mathrm{mmol} / \mathrm{L}$. Frequent monitoring of the patient's blood glucose should be initiated as per your ICU's usual practice for patients receiving an insulin infusion.

If insulin infusion is required at $\geq 6$ units/hr to maintain glucose target:

- Reduce Kabiven G19\% to $40 \mathrm{~mL} / \mathrm{hr}$ for 24 hours.

- At the end of 24 hours, if insulin needs are reduced below 6 units/hr, increase Kabiven G $19 \%$ to 80 mLs (or original goal rate, whichever is lower) for 24 hours.

- At the end of this second 24 hour period, if insulin needs remain below 6 units $/ \mathrm{hr}$, increase Kabiven GI9\% to goal rate.

- If insulin requirements exceed 6 units/hr at any time during the above process, reduce PN to previously tolerated rate, or $40 \mathrm{mLs} / \mathrm{hr}$ (whichever is higher), for 24 hours. Begin increasing rate every 24 hours as above, if tolerated. 
Table S2 (Continued)

Study PN Protocol B: malnourished early PN patients (ex BMI $\leq$ I7)

Feeding day I (first $24 \mathrm{hr}$ of PN)

- Commence Kabiven G19\% at $40 \mathrm{~mL} / \mathrm{hr}$ (or goal rate, whichever lower).

- Strongly recommend administering 100 mg thiamine, commencing at least 30 minutes prior to initiation of Kabiven GI9\% infusion, as clinically indicated as per product licensing indications.

- Recommend daily administration of other vitamins, minerals and trace elements, as clinically appropriate.

Feeding day 2 (second 24 hours of PN)

- Increase Kabiven GI9\% to $60 \mathrm{~mL} / \mathrm{hr}$ (or goal rate, whichever is lower).

- Recommend daily administration of vitamins, minerals and trace elements, as clinically appropriate.

Feeding day 3 (next 24 hours)

- Increase Kabiven G19\% to goal rate, as appropriate.

- Recommend daily administration of vitamins, minerals and trace elements, as clinically appropriate.

- Recommend trialing enteral/oral nutrition, if clinically appropriate.

- Once the patient tolerates $\geq 475 \mathrm{kcal} / \mathrm{day}$ EN, complete remainder of 24 hour Kabiven infusion and do not hang another bag.

- If patient tolerates any oral caloric intake from food, complete remainder of 24 hour Kabiven infusion and do not hang another bag. Feeding day 4 (next 24 hours) plus all additional days after day 4

- May switch to parenteral nutrition solution tailored to patient's specific clinical needs. Goals not to exceed $25-35 \mathrm{kcal} / \mathrm{kg}$ and I.0-1.5 g protein/kg.

- Strongly recommend addressing long term needs regarding trace elements, minerals and vitamins as clinically appropriate.

- Recommend trialing enteral/oral nutrition, if clinically appropriate.

- Once the patient tolerates $\geq 475 \mathrm{kcal} /$ day EN, complete remainder of 24 hour Kabiven infusion and do not hang another bag.

- If patient tolerates any oral caloric intake from food, complete remainder of 24 hour Kabiven infusion and do not hang another bag.

Insulin/glucose protocol: early PN patients

If glucose levels exceed $10 \mathrm{mmol} / \mathrm{L}$ an insulin infusion should be commenced and titrated to achieve peak serum glucose levels of $<10 \mathrm{mmol} / \mathrm{L}$.

Frequent monitoring of the patient's blood glucose should be initiated as per your ICU's usual practice for patients receiving an insulin infusion.

If insulin infusion is required at $\geq 6$ units/hr to maintain glucose target:

- Reduce Kabiven G19\% to $40 \mathrm{~mL} / \mathrm{hr}$ for 24 hours.

- At the end of 24 hours, if insulin needs are reduced below 6 units $/ \mathrm{hr}$, increase Kabiven G19\% to $80 \mathrm{mLs}$ (or original goal rate, whichever is lower) for 24 hours.

- At the end of this second 24 hour period, if insulin needs remain below 6 units/hr, increase Kabiven GI $9 \%$ to goal rate.

- If insulin requirements exceed 6 units/hr at any time during the above process, reduce PN to previously tolerated rate, or $40 \mathrm{mLs} / \mathrm{hr}$ (whichever is higher), for 24 hours. Begin increasing rate every 24 hours as above, if tolerated.

Abbreviations: kcals, kilocalories; EN, enteral; ICU, intensive care unit; BMI, body mass index.

\section{Publish your work in this journal}

ClinicoEconomics \& Outcomes Research is an international, peerreviewed open-access journal focusing on Health Technology Assessment, Pharmacoeconomics and Outcomes Research in the areas of diagnosis, medical devices, and clinical, surgical and pharmacological intervention. The economic impact of health policy and health systems organization also constitute important areas of coverage. The manuscript management system is completely online and includes a very quick and fair peer-review system, which is all easy to use. Visit http://www.dovepress.com/testimonials.php to read real quotes from published authors. 